\title{
Multiple oesophago-respiratory fistulae: sequelae of pulmonary tuberculosis in retroviral infection
}

\author{
Soo Fin Low ${ }^{1}$, MMed, Chai Soon $\underline{N g i u}^{2}$, MMed, MRCP, Erica Yee $\underline{\text { Hing }}{ }^{1}$, MMed, Norzailin $\underline{\text { Abu }} \underline{\text { Bakar }}{ }^{1}$, MMed
}

\begin{abstract}
Pulmonary tuberculosis (PTB) is a common infectious disease worldwide. However, mediastinal tuberculous lymphadenitis complicated by oesophageal involvement and oesophago-respiratory fistula is now uncommon due to improved anti-tuberculous regimes and better general awareness. The overall incidence of acquired oesophago-respiratory fistula due to infection is low, and therefore, the lesion is not often a frontrunner in differential diagnosis. Still, tuberculous oesophago-respiratory fistulae can potentially occur in patients with retroviral disease, as they tend to have atypical and more virulent manifestations. In this study, we report the case of multiple oesophago-respiratory fistulae in a patient with PTB and retroviral disease, and highlight the computed tomography features of these lesions as an atypical presentation of PTB in retroviral disease. Clinicians should suspect oesophago-respiratory fistulae if patients present with Ono's sign, and remain particularly vigilant for patients with underlying PTB and retroviral disease, as early diagnosis and treatment could help to reduce mortality.
\end{abstract}

Keywords: oesophago-bronchial fistula, oesophago-mediastinal fistula, oesophago-pulmonary fistula, oesophago-respiratory fistula, pulmonary tuberculosis

\section{INTRODUCTION}

Pulmonary tuberculosis (PTB) is a common infectious disease worldwide. With better antituberculous regimes and increased general awareness, mediastinal tuberculous lymphadenitis complicated by oesophageal involvement has now become uncommon. ${ }^{(1)}$ However, patients with retroviral disease tend to have atypical presentation and a more virulent course of the disease. ${ }^{(2)}$ They also have a higher tendency to develop tuberculous oesophago-respiratory fistulae. ${ }^{(3)}$ The incidence of oesophago-respiratory fistulae and tuberculosis in retroviral disease is also expected to be elevated. ${ }^{(4)}$ In this study, we report the case of multiple oesophago-respiratory fistulae in a patient with PTB and retroviral disease.

\section{CASE REPORT}

A 39-year-old man presented with shortness of breath for three months, with a two-month history of productive cough, fever and loss of appetite. He denied any history of haemoptysis. He was an intravenous drug user and was seropositive for retroviral disease, hepatitis B and hepatitis C. Physical examination revealed that the patient was cachexic, tachycardic (125 beats per min), tachypnoeic and febrile (body temperature, $38.4^{\circ} \mathrm{C}$ ), with an oxygen saturation of $94 \%$ on high flow mask. Chest auscultation revealed generalised reduced air entry, positive tactile fremitus and crepitation. Later, a chest radiograph showed a large left lung cavity with air fluid level and bilateral patchy air space opacities consistent with features of active PTB. Haematological investigations revealed pancytopenia, and markedly increased C-reactive protein level and erythrocyte sedimentation rate. The patient's renal profile and liver function test were unremarkable. Three sputum samples were positive for acid-fast bacilli. He was started on an antituberculous regime and intravenous ceftriaxone. However, the patient did not respond to medical therapy, with continued spiking of temperature and no oral tolerance. He experienced intractable cough and dyspnoea, particularly during feeding and drinking (suggestive of Ono's sign).

The patient's condition continued to deteriorate further, requiring mechanical ventilation support. Following intubation of the endotracheal tube, we noted a persistent gurgling sound originating from the patient's mouth upon application of positive pressure ventilation. A repeat laryngoscopy showed the correct placement of the endotracheal tube and functioning cuff. As the observation raised a suspicion of tracheo-oesophageal fistula, an urgent oesophagoscopy was performed. The oesophageal mucosa was found to be coated with confluent yellow-white plaques, signifying extensive candidiasis. A clean-edge oesophageal diverticulum $26 \mathrm{~cm}$ from the incisor, arising from the left lateral oesophageal wall, was also noted. A fistula opening was observed at the medial aspect of the diverticulum. However, further assessment of the oesophageal diverticulum (by passing the scope through it) was not attempted. A presumed diagnosis of oesophageal traction diverticulum with oesophago-bronchial fistula was made. Bronchoscopy was not performed in view of the patient's respiratory distress.

After oesophagoscopy, the patient developed multiple episodes of hypoxaemia. For this reason, computed tomography (CT) of the thorax was only performed four days after the procedure, when his condition had stabilised. CT of the thorax showed a large left lung cavity with air fluid level within (Figs. 1 \& 2). At the carinal level, a large oesophago-mediastinal fistula arising from the left lateral aspect of the oesophagus was seen. The fistula curved anteromedially and coursed the aortopulmonary window. Laterally, the fistula was communicating with the left lung cavity. Medially, there was another short and small fistula communicating between the left main bronchus

${ }^{1}$ Department of Radiology, ${ }^{2}$ Department of Medicine, Universiti Kebangsaan Malaysia Medical Centre, Kuala Lumpur, Malaysia

Correspondence: Dr Low Soo Fin, Radiologist, Department of Radiology, Universiti Kebangsaan Malaysia Medical Centre, Jalan Yaakob Latiff, 56000 Cheras, Kuala Lumpur, Malaysia.soofinlow@gmail.com 
and oesophago-mediastinal fistula. Multiple necrotic mediastinal lymphadenopathies were demonstrated. There were extensive consolidations and a tree-in-bud appearance, representing the classic presentation of active PTB.

The final diagnosis was oesophago-mediastinal fistula, oesophago-bronchial fistula and oesophago-pulmonary fistula secondary to active PTB. Oesophageal stenting was planned for the patient. However, one day after CT of the thorax, the patient developed massive haemoptysis; he coughed up two bouts of frank blood, with an estimated blood loss of $2 \mathrm{~L}$, and eventually succumbed to it.

\section{DISCUSSION}

Oesophago-respiratory fistula is an abnormal communication between the oesophagus and respiratory system, and can either be tracheal or via the bronchus or lung parenchyma. ${ }^{(5)}$ Benign oesophago-respiratory fistula in adults is rare, accounting for only $5.7 \%$ of the total acquired cases. ${ }^{(6)}$ The overall incidence of acquired oesophago-respiratory fistula due to infection is low, and therefore, it is often not a frontrunner in the differential diagnosis of such lesions. However, tuberculosis remains a leading cause of oesophago-respiratory fistula, particularly given its increased incidence in patients with retroviral disease. ${ }^{(7)}$ Generally, patients with retroviral infection tend to have atypical and more virulent manifestations. ${ }^{(2)}$

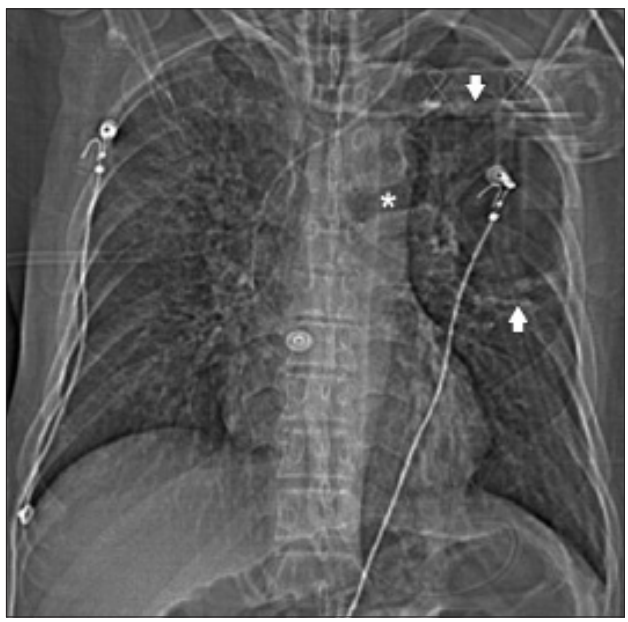

Fig. 1 CT image of the thorax shows a large cavity (arrowheads) in the left lung and a fistulous communication with the mediastinum (asterisk).
Oesophagitis is a common cause of morbidity in retroviral disease and Candida spp. is the most common causative organism. ${ }^{(8)}$ Other fungi, mycobacteria, viruses, bacteria or a combination of organisms may also potentially cause oesophagitis in retroviral disease. ${ }^{(8)}$ In our study, the patient was positive for not only retroviral disease with oesophageal candidiasis, but also mediastinal and pulmonary tuberculoses.

Several factors account for the development of oesophagorespiratory fistula. For one, caseating mediastinal tuberculous lymphadenitis tends to exert inflammatory reaction on adjacent structures. Tuberculous lymphadenitis may even adhere to the oesophagus, leading to either full-thickness erosion or traction of the oesophageal wall. ${ }^{(9)}$ The former would lead to an oesophagomediastinal fistula, while the latter would cause an oesophageal traction diverticulum. Apart from this, oesophagitis in retroviral disease may cause ulceration, perforation, fistula formation, haemorrhage and stricture of the oesophagus. ${ }^{(8)}$

On oesophagoscopy we found oesophageal candidiasis with a clean-edge, outpouching sac from the oesophagus, and thus postulated that the smooth and clean outpouching sac was most likely caused by the traction effect of tuberculous lymphadenitis. However, we did not advance the scope into the outpouching sac for further exploration, thus underestimating the length and extension of the lesion. The patient was consequently diagnosed with an oesophageal diverticulum and broncho-oesophageal fistula based on the initial findings of the oesophagoscopy; the oesophago-pulmonary fistula was detected only on CT of the thorax later. CT findings in our patient favoured oesophagomediastinal fistula rather than oesophageal diverticulum due to the length and curvilinearity of the tract. Conversely, it is also possible that the lesion was a traction oesophageal diverticulum that was subsequently eroded by the tuberculous nodes, resulting in multiple fistulae formation to the mediastinum, left main bronchus and left lung. By and large, histopathological examination is necessary to distinguish a diverticulum from a fistula.

Due to its anatomical location, the mid-oesophagus is in close proximity to the trachea and left main bronchus, and consequently, oesophago-mediastinal fistulae have mostly been reported to occur in the subcarinal region. ${ }^{(5)}$ In our patient, however, the fistula was seen at a slightly higher level (i.e. at the carinal level). Most case studies in the literature have reported
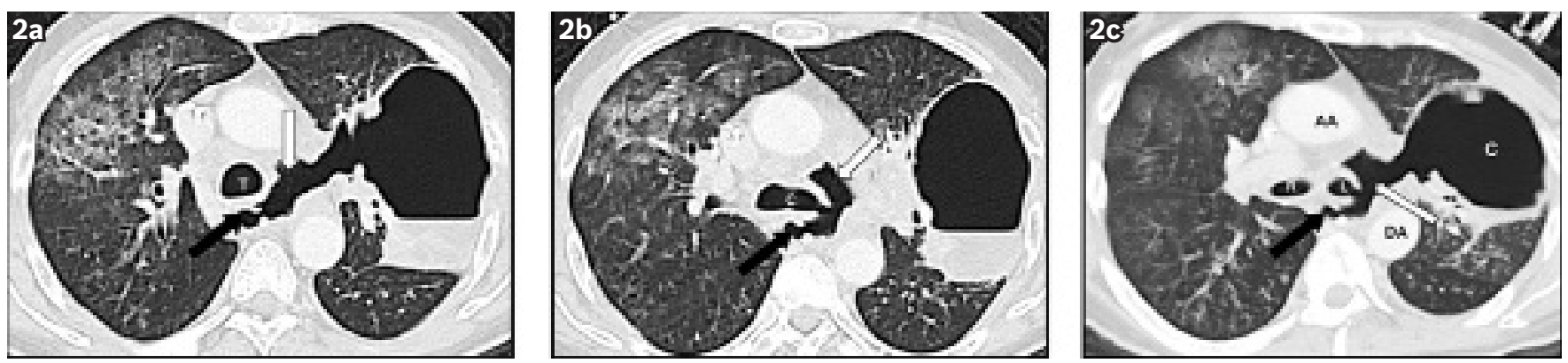

Fig. 2 Axial CT images of the thorax and lung window. (a) At the level just above the carina, the oesophago-mediastinal fistula is seen communicating with the left lung cavity (white arrow). (b) At the carinal level, the left main bronchus communicates with the oesophago-mediastinal fistula via a short fistula. (c) Axial oblique view shows all fistulae in a single slice, with a patchy consolidations in the right lung.

AA: ascending aorta; black arrow: oesophagus; white arrow: oesophago-mediastinal fistula; C: carina; DA: descending aorta; L: left main bronchus; R: right main bronchus; $\mathrm{T}$ : trachea 
patients with small and collapsed fistula tracts, ${ }^{(5,9,10)}$ whereas our patient had multiple large and long oesophago-respiratory fistulae.

Oesophago-respiratory fistulae are usually detected via oesophagoscopy and contrast oesophagogram. The morphology of mediastinal tuberculous lymphadenopathies and the course of oesophago-respiratory fistulae are generally well depicted by CT of the thorax. ${ }^{\left({ }^{8}\right)}$ Oesophagoscopy serves as both a diagnostic and therapeutic tool for these lesions. However, patients with oesophago-respiratory fistulae are generally ill and unable to tolerate any surgical correction. ${ }^{(11)}$ When medications alone fail to close the fistula in these patients, oesophageal prosthesis is considered the treatment of choice. ${ }^{(11)}$

In summary, we reported the case of a patient with PTB and retroviral disease who had multiple oesophago-respiratory fistulae, and highlighted the $\mathrm{CT}$ features of these lesions as an atypical presentation of РTB in retroviral disease. In patients presenting with Ono's sign, particularly those with underlying PTB and retroviral disease, clinicians should suspect oesophagorespiratory fistula. Early diagnosis and treatment could help to reduce the mortality rates in these patients.

\section{REFERENCES}

1. Yilmaz E, Akkoclu A, Sevinc C. CT and MRI appearance of a fistula between the right and left main bronchus caused by tracheobronchial tuberculosis. Br J Radiol 2001; 74:1056-8.

2. Daley CL, Small PM, Schecter GF, et al. An outbreak of tuberculosis with accelerated progression among persons infected with the human immunodeficiency virus. An analysis using restriction-fragment-length polymorphisms. N Engl J Med 1992; 326:231-5.

3. Alkhuja S, Miller A. Tuberculous bronchoesophageal fistulae in patients infected with the human immunodeficiency virus: a case report and review. Heart Lung 1998; 27:143-5.

4. Markowitz N, Hansen NI, Hopewell PC, et al. Incidence of tuberculosis in the United States among HIV-infected persons. The Pulmonary Complications of HIV Infection Study Group. Ann Intern Med 1997; 126:123-32

5. Ohtake M, Saito H, Okuno M, Yamamoto S, Ohgimi T. Esophagomediastinal fistula as a complication of tuberculous mediastinal lymphadenitis. Intern Med 1996; 35:984-6.

6. Mangi AA, Gaissert HA, Wright CD, et al. Benign broncho-esophageal fistula in the adult. Ann Thorac Surg 2002; 73:911-5.

7. Shah CP, Yeolekar ME, Pardiwala FK. Acquired tracheooesophageal fistula. J Postgrad Med 1994; 40:83-4.

8. Bianchi Porro G, Parente F. Opportunistic infections of the oesophagus in AIDS patients: clinical and therapeutic problems. J Intern Med 1993; 233:107-10.

9. Ballehaninna UK, Shaw JP, Brichkov I. Traction esophageal diverticulum: a rare cause of gastro-intestinal bleeding. Springerplus 2012; 1:50.

10. Rastogi A, Sarda D, Kothari P, Kulkarni B. Mediastinal tuberculosis presenting as traction diverticulum of the esophagus. Ann Thorac Med 2007; 2:126-7.

11. Temes RT, Wong RS, Davis M, Kessler RM, Wernly JA. Esophago-airway fistula in AIDS. Ann Thorac Surg 1995; 60:440-2. 